\title{
Revista Colombiana de

\section{Comportamiento de los parámetros hemodinámicos evaluados por el monitoreo ambulatorio de presión arterial de 24 horas}

\author{
María Gabriela García Orjuela a,*, César Caraballo Cordovez ${ }^{\mathrm{b}}$, \\ Andrea Hincapié Hincapié ${ }^{c}$, Esteban Prieto Bravoc, \\ Natalia Andrea Henao Sánchez ${ }^{a}$, Camilo Velásquez Mejía ${ }^{d}$, \\ Julián Zapata ${ }^{a}$, Ricardo Antonio Consuegra Peña ${ }^{a}$, Daniela Pastrana ${ }^{c}$, \\ Heidy Contreras ${ }^{\mathrm{a}}$ y Nicolás Jaramillo ${ }^{\mathrm{a}}$
}

a Centro de Medicina del Ejercicio y Rehabilitación Cardíaca CEMDE S.A., Medellín, Colombia

b Medicina Universidad de Antioquia, Medellín, Colombia

c Universidad CES., Medellín, Colombia

${ }^{\mathrm{d}}$ Facultad de Medicina, Universidad CES, Medellín, Colombia

Recibido el 12 de agosto de 2015; aceptado el 18 de abril de 2016

Disponible en Internet el 3 de junio de 2016

\section{PALABRAS CLAVE}

Presión arterial;

Hipertensión arterial;

Diagnóstico

\begin{abstract}
Resumen
Objetivo: Describir el comportamiento de los parámetros hemodinámicos en un grupo de individuos sometidos al estudio de monitoreo ambulatorio de la presión arterial.

Materiales y métodos: Estudio observacional descriptivo sobre el comportamiento de los parámetros hemodinámicos en el estudio de monitoreo ambulatorio de la presión arterial en un grupo de pacientes atendidos en dos instituciones de Medellín.

Resultados: Se analizaron un total de 108 pacientes atendidos en el período comprendido entre septiembre de 2012 y octubre de 2013. Los promedios de la presión arterial sistólica y presión arterial diastólica en 24 horas fueron de $128.34 \pm 12.79 \mathrm{mmHg}$ y $75.87 \pm 8.74 \mathrm{~mm} \mathrm{Hg}$, respectivamente. La media de la presión del pulso fue de $52.47 \pm 11.12 \mathrm{mmHg}$. Las presiones arteriales medias diurna y nocturna fueron de $96,23 \pm 9,5 \mathrm{mmHg}$ y $85.19 \pm 10,23 \mathrm{mmHg}$, respectivamente. La frecuencia cardiaca fue de $71.4 \pm 10.03$ latidos por minuto, para la carga sistólica la media fue de $39,38 \pm 27,80 \%$ y para la carga diastólica fue de $26,26 \pm 24,32 \%$.
\end{abstract}

\footnotetext{
is Este estudio fue aprobado por el Comité de Ética de la Investigación de CEMDE S.A. y siguió la normativa vigente expresada en la Declaración de Helsinki de la World Medical Association. Todos los participantes incluidos firmaron un consentimiento informado.

* Autor para correspondencia. Calle 33 A \# 70A-175. Teléfono: +4112421. fax: +4112352.

Correo electrónico: magabrielagarcia@yahoo.com (M.G. García Orjuela).
} 
Conclusiones: El monitoreo ambulatorio de la presión arterial es considerado actualmente como el método más confiable y exacto para el diagnóstico y evaluación de la hipertensión arterial. Permite no solo el diagnóstico y seguimiento del comportamiento de las cifras tensionales en los hipertensos, sino la evaluación de otras variables, como es mostrado en este estudio, con un comportamiento de los resultados muy similar a otros estudios locales.

(c) 2016 Sociedad Colombiana de Cardiología y Cirugía Cardiovascular. Publicado por Elsevier España, S.L.U. Este es un artículo Open Access bajo la licencia CC BY-NC-ND (http:// creativecommons.org/licenses/by-nc-nd/4.0/).

\section{KEYWORDS}

Blood pressure; Arterial hypertension; Diagnosis

\section{Introducción}

La hipertensión arterial es una patología de alta prevalencia, presentándose en un $40 \%$ en mayores de 25 años de edad, según la Organización Mundial de la Salud (OMS), y se estima que para el año 2025 sea del 60\% ${ }^{1}$. En Colombia, para el año 2008, según la Encuesta Nacional de Salud del Ministerio de Protección Social, se definió que en la población de 18 a 69 años, un $22.8 \%$ eran hipertensos ${ }^{2}$. Estas cifras son bastante significativas, ya que la hipertensión arterial es considerada un factor de riesgo cardiovascular para el desarrollo de las patologías como: la enfermedad cerebrovascular, la enfermedad coronaria y la enfermedad renal, entre otras.

Debido a lo anterior, se ha intentado diagnosticar la hipertensión arterial de una forma precoz para así tratarla oportuna y eficazmente evitando dichos desenlaces. Esto se ha ido logrando por medio del control y seguimiento de la presión arterial, desde la toma manual en el consultorio, hasta su toma en diferentes momentos del día con el monitoreo ambulatorio de la presión arterial (MAPA). Tradicionalmente la presión arterial se ha medido en el consultorio con la ayuda del estetoscopio y el esfigmomanómetro. No obstante, en los últimos tiempos, se ha propuesto utilizar equipos que lo hacen automáticamente, de una forma seriada, mientras el paciente está en el consultorio o en su hogar, en un ambiente tranquilo ${ }^{3}$.

Actualmente, existen muchas formas de medir la presión arterial, una de las técnicas más utilizadas es en la casa por los mismos pacientes con dispositivos automáticos ${ }^{4}$, inclusive algunos estudios sugieren, que es un mejor predictor para el riesgo cardiovascular las tomas hechas en la casa que en el consultorio ${ }^{3}$.

Sin embargo, las barreras socioeconómicas hacen más difícil estas tomas en la casa en cuanto a dispositivos; teniendo en cuenta también que durante el día pueden variar los valores, ya que influyen muchos factores como: el fumar, la ingesta de cafeína, el ejercicio, entre otros ${ }^{5}$; lo que debe ser considerado a la hora de interpretarlos.

El MAPA ha sido ampliamente estudiado y es de mucho interés científico en los últimos años, con más de 10.000 publicaciones relacionadas en Pubmed en el año 2012 y considerado como el estándar de oro para el diagnóstico de la hipertensión arterial, según las guías del National Institute for Health and Clinical Excellence (NICE) en el Reino Unido, publicadas en el año $2011^{3}$. Esta técnica utiliza un dispositivo que mide la presión arterial en un período que varía de 24 a 48 horas. Normalmente cada 15-20 minutos durante el día 
y cada 30-60 minutos durante el sueño. Estas cifras se graban en el dispositivo, para luego ser evaluadas y mediante un computador realizar los promedios de la presión arterial que se obtuvieron en el día y en la noche ${ }^{4}$. Además, de evaluar las cifras de la presión arterial, el MAPA permite analizar otras variables hemodinámicas, como: la frecuencia cardiaca, el fenómeno dipper, las cargas sistólicas y diastólicas, y la presión de pulso; las cuales se han relacionado con mayor morbimortalidad ${ }^{6}$.

En Colombia son pocos los estudios que se han desarrollado del MAPA como herramienta de diagnóstico y seguimiento de la hipertensión arterial, es así, el caso de un estudio realizado en la ciudad de Cali en el año 2004, en el cual se buscó como objetivo determinar los factores asociados al no control de la presión arterial; se concluyó que un $30 \%$ de la población evaluada no tenía un adecuado control siendo mayor en el género masculino, con un adherencia del $56 \%$ al tratamiento farmacológico, distinguiendo como factores asociados al mal control de la presión arterial al tipo de la entidad prestadora de salud, el género masculino, el uso de los inhibidores de la enzima convertidora de angiotensina y la interacción del medicamento con antecedentes de dislipidemia ${ }^{7}$. Recientemente, fueron publicados dos estudios realizados en la ciudad de Medellín. En el primero realizado por Mónica et al., se comparó la toma seriada de la presión arterial en el consultorio con la monitorización arterial durante 24 horas para el diagnóstico ambulatorio de la hipertensión arterial, encontrándose que la toma seriada de la presión arterial comparada con la monitorización ambulatoria de la misma, tiene alta sensibilidad (mayor de $88 \%$ ) y baja especificidad (menor de $22 \%$ ) y no es recomendable como prueba de referencia ${ }^{1}$. El segundo estudio a su vez, liderado por Patiño S. y Cols., buscaba establecer una correlación entre la medición de la presión arterial en el consultorio y el MAPA en pacientes con diagnóstico reciente de la hipertensión arterial, en este estudio se encontró que existe una correlación positiva entre la medición de la presión arterial clínica y por la MAPA, pero la sensibilidad y la especificidad de una sola medición en el consultorio con respecto al MAPA, la hace insuficiente para verificar el control de la presión arterial $^{8}$. De ambos estudios se concluye de que a pesar de que existe una correlación positiva entre la medición de la presión arterial clínica y por el MAPA, la sensibilidad y la especificidad de la primera es insuficiente para el diagnóstico y el seguimiento del control de la presión arterial con respecto al monitoreo ambulatorio de la presión arterial.

Una de las limitaciones importantes en la realización del estudio MAPA es la pobre tolerancia a la realización de este examen, generando incomodidad durante la toma de las presiones, las cuales, al ser frecuentes durante el día, pueden interferir con el bienestar y sueño de los pacientes. Es por esto que se evaluó la intolerancia en este estudio a través del diseño de una encuesta sencilla diligenciada por el paciente al momento de finalizar el estudio.

En el presente estudio nuestro grupo buscó describir el comportamiento de los parámetros hemodinámicos evaluados por el MAPA y la caracterización de la población medida por este tipo de instrumento, en dos instituciones prestadoras de salud de la ciudad de Medellín.

\section{Métodos y materiales}

\section{Tipo de estudio y población}

Se realizó un estudio observacional descriptivo sobre el comportamiento de los parámetros hemodinámicos en un grupo de pacientes a quienes se les realizó el estudio de monitoreo ambulatorio de la presión arterial (MAPA) en el Centro de Medicina del Ejercicio y Rehabilitación Cardiaca (CEMDE S.A.) y el Centro Cardioestudio; ambos ubicados en la ciudad de Medellín (Colombia), entre septiembre de 2012 y octubre de 2013.

Solo se incluyeron pacientes mayores de edad y se excluyeron aquellos con diagnóstico de hipertensión arterial secundaria o hipertensión arterial asociada al embarazo. Todos los participantes firmaron consentimiento informado.

Se utilizó un equipo marca Suntech, modelo 222 Bravo, serial 6759, previamente calibrado. A los pacientes se les puso el monitor en su brazo no dominante y se grabaron datos durante 24 horas, realizando tomas diurnas cada 20 minutos y nocturnas cada 30 minutos con límites normales de la presión arterial diurna de $135 / 85 \mathrm{mmHg}$ y nocturna de $120 / 80 \mathrm{mmHg}$. A los pacientes se les indicó hacer las actividades de rutina y se les entregó un diario para registrar dichas actividades y síntomas presentados durante el estudio. Al momento del retiro del equipo, se les aplicó una encuesta sobre la tolerancia al dispositivo. Se les preguntaba sobre la incomodidad que les ocasionó la prueba, pidiéndoles que lo clasificaran así: intolerancia severa (imposible aguantar la molestia de las tomas seriadas en todo momento); moderada intolerancia (molestias en ciertas circunstancias pero que no impiden realizarse el estudio); y mínima intolerancia (molestias que no generan intolerancia en ningún momento del estudio).

La información se tabuló en el software MS Office Excel ${ }^{\circledR}$ 2010 y su interpretación fue realizada por los médicos del estudio, quienes verificaron previamente la validez de los datos captados y grabados, constituyéndose como un examen válido todo aquel que registre por lo menos 50 tomas válidas o que mínimamente lleguen al $70 \%$ del total de las tomas ${ }^{3}$.

Los parámetros utilizados en el la medición de las variables del estudio MAPA son ${ }^{9,10}$ :

- Presión arterial: valores normales menores de $135 / 85 \mathrm{mmHg}$ en el período diurno, $120 / 80 \mathrm{mmHg}$ en el período nocturno, $130 / 80 \mathrm{mmHg}$ en el período comprendido en las 24 horas.

- Cargas hipertensivas: valores normales menor del 20\%, prehipertensión arterial: entre el $20 \%$ y el $30 \%$ y altas: mayor del $30 \%$.

- Altas cargas sistólicas: considerándose como carga hipertensiva sistólica mayor del 30\%.

- Altas cargas diastólicas: considerándose como carga hipertensiva diastólica mayor de $30 \%$.

- Hipertensión arterial: se considera controlada con cargas hipertensivas menores del $30 \%$ y no controlada con cargas mayor o igual al 30\%. Hipertensión arterial de novo se consideró el tener un promedio de presión arterial por encima de los valores definidos sumado a la presencia de altas cargas sistólicas o diastólicas. 
- Hipotensión arterial: definiéndose de acuerdo a la variación en el día así. Período diurno, menor de valores de $98 / 61 \mathrm{mmHg}$ en las mujeres y menor de valores de $110 / 65 \mathrm{mmHg}$ en los hombres y período nocturno, menor de valores de $85 / 45 \mathrm{mmHg}$ en ambos sexos.

- Fenómeno dipper: según la fórmula lo consideramos normal entre el $10 \%$ y el $20 \%$. Para este estudio, se consideró que valores menores al $10 \%$ o mayores al $20 \%$ como fenómeno no dipper.

- Frecuencia cardiaca: normal de 60 a 90 latidos por minuto, frecuencia baja con valores menores de 60 y frecuencia alta con más de 90 latidos por minuto.

- Presión de pulso: considerada como la diferencia entre la PAS y la PAD (PAS-PAD); se considera normal valores menores o iguales a $50 \mathrm{mmHg}$ y elevados aquellos valores mayores de $50 \mathrm{mmHg}$.

Determinación del fenómeno dipper, calculándose con la fórmula:

Dipper : $\frac{\text { Presión arterial media diurna-presión arterial media nocturna }}{\text { Presión arterial media diurna }} \times 100$

Definiéndose Fenómeno dipper normal: Valores entre $10-20 \%$, clasificando como no-dipper a valores por fuera de ese rango.

Este estudio fue aprobado por el Comité de Ética de la Investigación de CEMDE S.A. y siguió la normativa vigente expresada en la Declaración de Helsinki de la World Medical Association. Todos los participantes incluidos firmaron un consentimiento informado.

\section{Análisis estadístico}

La información se digitalizó en una hoja de cálculo de MS Office Excel ${ }^{\circledR} 2010$, luego se analizó en el programa estadístico PASW Statistics $18^{\circledR}$ (SPSS 18). Las tablas, los gráficos y la presentación textual, se realizó mediante el procesador de texto de MS Office Word $^{\circledR} 2010$.

Por tratarse de una descripción de un grupo de pacientes no se calculó el tamaño de la muestra.

Se utilizó la prueba de normalidad para evaluar si la distribución de las variables cuantitativas se ajustaba a una distribución normal. Para las variables cuantitativas con distribución normal, se utilizaron el promedio y la desviación estándar (DE), en caso de no distribuir normal la mediana y el rango intercuartil.

Las variables cualitativas se presentan en valores absolutos y relativos.

Se compararon los grupos utilizando la prueba $\chi 2$ de independencia para determinar si existían diferencias entre los pacientes que presentaban o no alteración en el Dipper, comparados con los pacientes que tenían o no HTA controlada, presión de pulso normal o anormal. Se utilizó un $\alpha$ de 0,05 y $p<0,05$ como valor significativo.

\section{Resultados}

Se revisaron los registros de un total de 117 pacientes a quienes se les hizo el MAPA en el período comprendido entre septiembre de 2012 y octubre de 2013, de los cuales
9 pacientes no fueron incluidos, 5 de ellos por no tener el consentimiento informado y 4 por ser menores de edad. Finalmente se incluyeron un total de 108 pacientes.

Las características demográficas se describen en la Tabla 1. La media de la edad fue de 53.56 DE -15.87, con una edad mínima de 18 y una máxima de $87,52.8 \%$ pertenecían al género femenino.

Se encontró que el promedio de la presión arterial sistólica en 24 horas fue de $128.34 \pm 12.79 \mathrm{mmHg}$, para la presión arterial diastólica el promedio fue de $75.87 \pm 8.74 \mathrm{mmHg}$, para la presión de pulso los valores fueron $52.47 \pm 11.12 \mathrm{mmHg}$, para la presión arterial media diurna fue de $96,23 \pm 9,5 \mathrm{mmHg}$, para la presión arterial media nocturna fue de $85.19 \pm 10,23 \mathrm{mmHg}$, la frecuencia cardiaca fue de $71.4 \pm 10.03$ latidos por minuto, para la carga sistólica fue de $39,38 \pm 27,80 \mathrm{~mm} \mathrm{Hg}$ y para carga diastólica fue de $26,26 \pm 24,32 \mathrm{mmHg}$. Tabla 2 .

Al analizar el comportamiento de las cifras de la presión arterial se encontró que un $43,5 \%$ de los pacientes presentaban cifras por encima de los rangos establecidos como normales, con una presión sistólica máxima de $174 \mathrm{mmHg}$ y mínima de $101 \mathrm{mmHg}$, así mismo, una presión diastólica máxima de $106 \mathrm{mmHg}$ y mínima de $58 \mathrm{mmHg}$. El 70.4\% de los pacientes tenían antecedente ya conocido de hipertensión arterial, con el $61.8 \%$ de estos pacientes con mal control.

En cuanto al dipper se encontró que el $41.7 \%$ de los pacientes tenía un valor entre $10 \%$ y el $20 \%$ determinado como normal.

Con respecto a la intolerancia al dispositivo para el MAPA, se encontró moderada en el $60.2 \%$, mínima del $35,2 \%$ y grave solo en un 2,8\% de los pacientes de la población evaluada.

Al comparar las variables hipertensión arterial controlada o no con el dipper normal o alterado, no se encontraron diferencias estadísticas significativas (Prueba de $\mathrm{Chi}^{2}$ de independencia $p=0,522$ ), igualmente, se comparó la variable dipper normal o alterado con la presión de pulso sin encontrarse diferencias estadísticas (Prueba de $\mathrm{Chi}^{2}$ de independencia $\mathrm{p}=0,320$ ). Tabla 3 .

Se realizó una comparación de las variables hemodinámicas entre los pacientes con antecedente conocido de hipertensión arterial $(n=76)$ y los pacientes a quienes se les diagnóstico la hipertensión arterial durante el estudio $(n=11)$, sin encontrarse diferencias significativas. Dicha comparación se muestra en la Tabla 4.

Tabla 1 Características sociodemográficas

\begin{tabular}{ll}
\hline Sexo & $\mathrm{n}(\%)$ \\
\hline Femenino & $57(52,8 \%)$ \\
Edad $^{*}$ & Años \\
Media & 53,56 \\
DE & 15,877 \\
Grupos de edad & $\mathrm{n}(\%)$ \\
De 18 a 29 años & $9(8,3)$ \\
De 30 a 44 años & $21(19,4)$ \\
De 45 a 49 años & $41(38)$ \\
De 60 a 69 años & $19(17,6)$ \\
De 70 a 79 años & $10(9,3)$ \\
Mayores de 80 años & $8(7,4)$ \\
\hline * Media (x), desviación estándar (DE), Mediana (Me)
\end{tabular}


Tabla 2 Características hemodinámicas

\begin{tabular}{lccccccccc}
\hline & $\begin{array}{l}\text { PAS } \\
\mathrm{mmHg}\end{array}$ & $\begin{array}{l}\text { PAD } \\
\mathrm{mmHg}\end{array}$ & $\begin{array}{l}\text { Presión } \\
\text { de pulso }\end{array}$ & $\begin{array}{l}\text { PAM diurna } \\
\mathrm{mmHg}\end{array}$ & $\begin{array}{l}\text { PAM nocturna } \\
\mathrm{mmHg}\end{array}$ & $\begin{array}{l}\text { Valor } \\
\text { Dipper } \\
(\%)\end{array}$ & $\begin{array}{l}\text { FC } \\
\text { Latidos por } \\
\text { minuto }\end{array}$ & $\begin{array}{l}\text { Carga } \\
\text { Sistólica } \\
(\%)\end{array}$ & $\begin{array}{l}\text { Carga } \\
\text { Diastólica } \\
(\%)\end{array}$ \\
\hline $\begin{array}{l}\text { Media } \\
\text { Desviación }\end{array}$ & 128,34 & 75,87 & 52,47 & 96,23 & 85,19 & 10,49 & 71,4 & 39,38 \\
$\quad$ estándar & 12,79 & 8,74 & 11,12 & 9,5 & 10,23 & 8,64 & 10,03 & 27,80 & 24,32 \\
Mediana & 127 & 76,5 & 50,5 & 96 & 86 & 11,05 & 71 & 33,95 & 20,65 \\
Rq & 14 & 13 & 11 & 13 & 11 & 12,58 & 16 & 44 & 40,67 \\
Máximo & 174 & 106 & 107 & 123 & 125 & 26,13 & 94 & 100 & 100 \\
Mínimo & 101 & 58 & 31 & 75 & 63 & -10 & 42 & 0 \\
\hline
\end{tabular}

" Rango inter cuartil: Rq

Tabla 3 Variables clínicas de los pacientes

\begin{tabular}{|c|c|c|c|c|}
\hline \multirow[t]{2}{*}{ Variable } & \multicolumn{2}{|c|}{ DIPPER } & \multirow{2}{*}{$\begin{array}{l}\text { Total } \\
\text { n (\%) }\end{array}$} & \multirow[t]{2}{*}{ Valor $p$} \\
\hline & $\begin{array}{l}\text { Normal } \\
\mathrm{n}(\%)\end{array}$ & $\begin{array}{l}\text { Alterado } \\
\mathrm{n}(\%)\end{array}$ & & \\
\hline \multicolumn{5}{|l|}{ HTA } \\
\hline Controlada & $11(10,2)$ & $18(16,6)$ & $29(26,8)$ & 0,522 \\
\hline No controlada & $18(16,6)$ & $29(26,8)$ & $47(43,5)$ & \\
\hline No hipertensos & $16(14,8)$ & $16(14,8)$ & $32(29,6)$ & \\
\hline \multicolumn{5}{|l|}{ Presión de Pulso } \\
\hline Normal & $2(1,8)$ & $6(5,5)$ & $8(7,4)$ & 0,320 \\
\hline Anormal & $43(39,8)$ & $57(52,7)$ & $100(92,5)$ & \\
\hline \multicolumn{5}{|l|}{ Carga Sistólica } \\
\hline Controlada & $23(21,3)$ & $34(31,5)$ & $57(52,8)$ & 0,553 \\
\hline No controlada & $22(20,3)$ & $29(26,85)$ & $51(47,2)$ & \\
\hline \multicolumn{5}{|l|}{ Carga Diastólica } \\
\hline Controlada & $17(15,7)$ & $24(22,2)$ & $41(37,9)$ & 0,973 \\
\hline No controlada & $28(25,9)$ & $39(36,1)$ & $67(62,1)$ & \\
\hline \multicolumn{5}{|l|}{ Intolerancia } \\
\hline Mínima & $17(14,6)$ & $22(18,8)$ & $39(33,3)$ & 0,996 \\
\hline Moderada & $31(26,5)$ & $40(34,2)$ & $71(60,7)$ & \\
\hline Severa & $1(0,85)$ & $1(0,85)$ & $2(1,7)$ & \\
\hline
\end{tabular}

${ }^{*} \mathrm{Chi}^{2}$ de independencia, Significancia estadística $\mathrm{p}<0,05$

\section{Discusión}

Actualmente, el monitoreo ambulatorio de la presión arterial (MAPA) es el examen recomendado para el diagnóstico y seguimiento de los pacientes con hipertensión arterial ${ }^{3,11}$, permitiendo evaluar, no solo, las variaciones de la presión arterial tanto en el día como en la noche, incluso también otros parámetros como son la presión de pulso, frecuencia cardiaca y el fenómeno dipper ${ }^{3,12,13}$.

Las últimas guías para el manejo de la hipertensión arterial primaria en adultos del Instituto Nacional para la Salud y la Excelencia Clínica (del inglés, National Institue for Health and Clinical Excellence (NICE)) recomienda al MAPA como la herramienta para la confirmación del diagnóstico de la hipertensión arterial ${ }^{9,14}$. Por otra parte, las últimas guías la Sociedad Europea de Hipertensión (del inglés, European Society of Hypertension (ESH)) y la Sociedad Europea de
Cardiología (del inglés, European Society of Cardiology (ESC)), consideran al MAPA como una herramienta importante para el diagnóstico y seguimiento de la hipertensión arterial $^{3,14}$. En la actualidad, varios autores están recomendando al MAPA no solo como la herramienta diagnóstica sino también como un medio para realizar el seguimiento de la enfermedad y evaluar la efectividad del tratamiento farmacológico debido a que no solo evalúa las cifras tensionales, sino otras variables como son el fenómeno dipper, la presión de pulso, y frecuencia cardiaca, cuyas alteraciones se han relacionado con el aumento del riesgo cardiovascular ${ }^{14,15}$.

Es sabido que los pacientes, tanto hipertensos como normotensos, que presentan una disminución del dipper $(<10 \%)$ durante la noche, conocidos como pacientes no-dippers, tienen mayor riesgo de presentar complicaciones micro y macrovasculares $8,13,16$. Así como también una caída exagerada (> 20\%) del dipper, está asociada a eventos coronarios y 
Tabla 4 Análisis por subgrupos de pacientes con diagnóstico conocido de hipertensión arterial vs. diagnóstico de Novo

\begin{tabular}{|c|c|c|c|c|}
\hline \multirow[t]{2}{*}{ Variable } & \multicolumn{2}{|c|}{ Hipertensión arterial } & \multirow{2}{*}{$\begin{array}{l}\text { Total } \\
\mathrm{n}(\%)\end{array}$} & \multirow[t]{2}{*}{ Valor $p$} \\
\hline & $\begin{array}{l}\text { Antecedente conocido } \\
\mathrm{n}=76(\%)\end{array}$ & $\begin{array}{l}\text { De Novo } \\
n=11(\%)\end{array}$ & & \\
\hline Presión pulso normal (< 50 mmHg) & $37(43)$ & $3(3)$ & $40(46)$ & 0,313 \\
\hline Dipper normal (10-30\%) & $30(34)$ & $5(6)$ & $35(40)$ & 0,961 \\
\hline Promedio frecuencia cardiaca normal (60-90 lpm) & $62(71)$ & $11(13)$ & $73(84)$ & 0,265 \\
\hline Carga sistólica normal (< 30\%) & $29(33)$ & $1(1)$ & $30(34)$ & 0,12 \\
\hline Carga diastólica normal (<30\%) & $44(51)$ & $8(9)$ & $52(60)$ & 0,543 \\
\hline PAM normal $(70-110 \mathrm{mmHg})$ & $72(83)$ & $11(13)$ & $83(95)$ & 0,993 \\
\hline
\end{tabular}

cerebrovasculares, por exposición a bajas perfusiones arteriales, estando esta disminución exagerada, estrechamente relacionada con un aumento excesivo de la presión arterial en la mañana ${ }^{3,12,13}$. En nuestro estudio encontramos que el $41.7 \%$ de todos los pacientes evaluados, independientemente, de tener diagnóstico o no de la hipertensión arterial, presentaron el fenómeno dipper dentro de los rangos normales.

A su vez, la elevación de la presión del pulso se ha utilizado como marcador indirecto de la rigidez arterial, la cual es considerada un factor de riesgo para eventos coronarios $^{16,17}$. En nuestro estudio se encontró la presión del pulso en promedio de $52.47 \mathrm{mmHg}$, sin ser un resultado estadísticamente significativo.

En el presente estudio, se incluyeron pacientes sin antecedente previo de la hipertensión arterial, considerándose como hipertensión de Novo, así como pacientes con diagnóstico ya establecido, estos últimos correspondiendo al 70.4\% ( $n=87)$ del total de pacientes. En general, sólo el 41.7\% tuvo una disminución normal del dipper (disminución del $10-20 \%)$. Esto estando en relación con estudios previos, como el publicado por la Sociedad Española de Hipertensión en el año 2007, donde se incluyeron 6.534 pacientes, de los cuales el $58,7 \%$ tenían un patrón de no-dipper ${ }^{18}$. Igualmente, al evaluar los pacientes con la hipertensión arterial establecida, se encontró que el $61.8 \%$ no estaban bien controlados, y al momento de analizar estos datos junto con la presión de pulso y las cargas tanto sistólica como diastólicas, no hubo una diferencia significativa.

El estudio MAPEC fue diseñado específicamente para comprobar la hipótesis de que la cronoterapia con uno o más medicamentos antihipertensivos antes de acostarse ejerce mejor control de la presión arterial y reducción del riesgo cardiovascular que la terapia convencional al levantarse. Este estudio demostró una reducción significativa en la prevalencia de no dipper en quienes recibieron medicación antes de acostarse. Fue el primer estudio en demostrar una reducción en los desenlaces clínicos, lo que sugiere este tipo de resultados, es que se debe tener presente las horas de toma de los diferentes medicamentos en los tratamientos antihipertensivos, como alternativa estratégica ${ }^{19}$.

Son pocos los estudios existentes que correlacionan la frecuencia cardiaca y la toma ambulatoria de la presión arterial utilizando el MAPA. La base de datos IDACO (International Database in Relation to Cardiovascular Outcome) se estableció en el año 2006, la cual incluye estudios que analizan los desenlaces cardiovasculares relacionados con los parámetros evaluados por el MAPA, incluyendo 12.148 pacientes de cohortes de Dinamarca, Bélgica, Japón, Suecia, Polonia, Uruguay, China, entre otros ${ }^{20,21}$. Dentro de los análisis realizados, se tomaron los datos de frecuencia cardiaca en el MAPA de 6.928 sujetos sin tratamiento con betabloqueadores con seguimiento a 9,6 años, encontrándose que la elevación de la frecuencia cardiaca estuvo relacionada con mayor mortalidad de causa no cardiovascular ${ }^{22}$. Dentro los resultados encontrados en el presente estudio, la frecuencia cardiaca permaneció dentro de rangos esperados con una media de $71.4 \pm 10.03$.

La rigidez arterial se ha considerado igualmente un predictor de desenlaces cardiovasculares adversos, siendo el método ideal para su medición la velocidad de onda de pulso. No obstante, debido a que se requiere de equipos especializados para su medición, se han recurrido a otros parámetros que ayudan a su evaluación de manera indirecta. En el caso del MAPA, se ha demostrado que la presión de pulso, definida como la diferencia entre la presión sistólica y la presión diastólica, se correlaciona de manera significativa con la rigidez arterial ${ }^{23}$. La alteración de este parámetro en pacientes con la hipertensión arterial, se ha asociado con daño de órgano blanco y con alteración de la tasa de filtración glomerular así como con eventos cardiocerebrovasculares ${ }^{23}$. Un estudio previo encontró que en pacientes hipertensos no tratados, la rigidez arterial fue más alta en dippers que en no dippers, e inversamente relacionada con los descensos nocturnos de la presión arterial ${ }^{24}$. Otro estudio determinó que la variabilidad de la presión de pulso, de la presión arterial sistólica y diastólica, así como el dipper, son predictores independientes de la rigidez arterial, debido a que se considera que reflejan el grosor de la íntima arterial ${ }^{25}$.

Dentro de las principales limitantes del estudio MAPA está la difícil tolerancia de los pacientes a las mediciones frecuentes de la presión arterial, ya que cada 20 - 30 minutos, el brazalete es insuflado, generando incomodidades, principalmente en la noche ${ }^{12}$. Es por esta razón que se evaluó el grado de intolerancia a la realización de este examen a través de una encuesta realizada a los pacientes, donde cada uno calificó la intolerancia según el grado de incomodidad sentido durante el estudio. Se encontró que la intolerancia calificada como moderada fue la que predominó, referida por el $60.2 \%$ de los pacientes, mientras el 34,3\% refirió mínima intolerancia y solo un $2 \%$ de los pacientes la calificó como grave. Este 
tipo de percepción de los pacientes es fundamental, para ser tenido en cuenta por el médico, cuando decide usar un instrumento o método diagnóstico.

Al momento de realizar cruces entre las variables, como: el valor de dipper y la intolerancia, el valor de dipper y el control de la hipertensión arterial, y la intolerancia con el control de la hipertensión arterial, no se encontraron diferencias significativas. Sin embargo, este estudio observacional sirve de base para nuevos interrogantes acerca de los distintos factores que pueden influenciar el comportamiento del fenómeno dipper, así como en el control de la hipertensión arterial, generando esto retos para estudios futuros.

\section{Conclusiones}

El monitoreo ambulatorio de la presión arterial (MAPA), es considerado actualmente el método más confiable y exacto para el diagnóstico y la evaluación de la hipertensión arterial, permitiendo no solo el evaluar las cifras tensionales sino el comportamiento del fenómeno dipper, presión de pulso y frecuencia cardiaca, los cuales se han relacionado con riesgo de complicaciones micro y macrovasculares así como con la mortalidad cardiovascular. Lo anterior no solo encontrado en el presente estudio sino también en estudios previos realizados localmente, con resultados similares.

La percepción y la tolerancia de este tipo de examen se ubican entre los métodos diagnósticos moderadamente molestos, sin causar un rechazo importante en la población en general.

Si bien está reportada en la literatura la pobre tolerancia al MAPA, no existen estudios que pretendan relacionar la influencia de la tolerancia con la mayor presentación de patrones no dipper. Dentro de las principales molestias reportadas se encuentran: la perturbación del sueño y/o la vida habitual de los pacientes e interferencia de la actividad física con las mediciones. Vale la pena desarrollar este tema en futuras investigaciones, para determinar hasta qué punto la tolerancia del método, pueda influir en los resultados básicos.

\section{Responsabilidades éticas}

Protección de personas y animales. Los autores declaran que los procedimientos seguidos se conformaron a las normas éticas del comité de experimentación humana responsable y de acuerdo con la Asociación Médica Mundial y la Declaración de Helsinki.

Confidencialidad de los datos. Los autores declaran que en este artículo no aparecen datos de pacientes

Derecho a la privacidad y consentimiento informado. Los autores han obtenido el consentimiento informado de los pacientes y/o sujetos referidos en el artículo. Este documento obra en poder del autor de correspondencia.

\section{Declaración de conflicto de intereses}

Los autores declaran que no hay conflicto de intereses

\section{Agradecimientos}

Agradecimientos al Centro de Medicina del Ejercicio y Rehabilitación Cardíaca (CEMDE S.A.), y las IPS y EPS que ayudaron a la realización de este estudio

\section{Bibliografía}

1. Giraldo M, Ibero G, García H. Comparación de la toma seriada de presión arterial y la monitorización ambulatoria para el diagnóstico de hipertensión esencial en una población colombiana. Rev Col Cardiol. 2013;20(6):342-51.

2. Ministerio de Protección Social Encuesta Nacional de Salud de Colombia. Bogotá: Ministerio de Protección Social; 2008.

3. European Society of Hypertension position paper on Ambulatory Blood Pressure Monitoring. J Hypertension. 2013;31: 1731-68.

4. Kaplan N, Bakris G, Gersh B. Ambulatory blood pressure monitoring and white coat hypertension in adults. Up to Date. Marzo. 2015.

5. Mann J, Bakris G, Kaplan N. Choice of drug therapy in primary (essential) hypertension: Recommendations. Up to date. Enero. 2015.

6. Sandoya E. Estado actual del monitoreo ambulatorio de presión arterial. Rev Urug Cardiol. 2013;28(2):273-84.

7. Herrera R, Badiel M, Zapata H. Factores asociados al no control de la presión arterial en pacientes inscritos al programa de hipertensión de una Entidad Promotora de Salud en CaliColombia, 2004. Rev Col Cardiol. 2009;16(4):143-52.

8. Patiño S, Toro J, Jaramillo C, García H, Giraldo M. Correlación entre la medición en consultorio y la monitorización ambulatoria de la presión arterial en pacientes hipertensos de Medellín, Colombia. Rev Col Cardiol. 2013;20(4):190-7.

9. National Institute for Health and Care Excellence. Clinical Management of Primary Hypertension in Adults. London: National Institute for Health and Care Excellence; 2011.

10. JCS Joint Working Group. Guidelines for the Clinical Use of 24 Hour Ambulatory Blood Pressure Monitoring (ABPM). Circ J. 2012;76(2):508-19.

11. Hamada T, Murata T, Narita K, Takahashi T, Wada Y, Kimura H, Yoshida $\mathrm{H}$. The clinical significance of abnormal diurnal blood pressure variation in healthy late middle-aged and older adults. Blood Pressure. 2008;17:134-40.

12. Turner JR, Viera AJ, Shimbo D. Ambulatory Blood Pressure Monitoring in Clinical Practice: A Review. Am J Med. 2015;128: 14-20.

13. Redon J. The Importance of 24-Hour Ambulatory Blood Pressure Monitoring in Patients at Risk of Cardiovascular Events. High Blood Press Cardiovasc Prev. 2013;20:13-8.

14. Hermida R, Smolensky M, Ayala D, Portaluppi F. Ambulatory Blood Pressure Monitoring (ABPM) as the reference standard for diagnosis of hypertension and assessment of vascular risk in adult. Chronobiol Int. 2015; Early Online:1-14.

15. Japanese Circulation Society Guidelines for the Clinical Use of 24 Hour Ambulatory Blood Pressure Monitoring (ABPM) (JCS 2010). Circ J. 2012;76:508-19.

16. Izzedine H, Launay-Vacher V, Deray G. Abnormal blood pressure circadian rhythm: A target organ damage? Int J Cardiol. 2006;107:343-434.

17. Makhoul N, Arraf Z, Khamisy-Farah R. Switching therapy to bedtime for uncontrolled hypertension with a nondipping pattern: a prospective randomized-controlled study. Blood Press Monit. 2013;18(4):227-31.

18. Bouhanick B, Chamontin B. Should pulse pressure and day/night variatons in blood pressure be seen as independent risk factors requiring correction or simply as markers to be taken into 
account when evaluating overall vascular risk? Diabetes Metab. 2007;33:321-30.

19. Mahabala C, Kamath P, Bhaskaran U, Pai ND, Pai AU. Antihypertensive therapy: nocturnal dippers and nondippers. Do we treat them differently? Vascular Health and Risk Management. 2013;9:125-33.

20. Gorostidi M, Sobrino J, Segura J, Sierra C, De la Sierra A, Hernández del Rey R, et al. Ambulatory blood pressure monitoring in hypertensive patients with high cardiovascular risk: a cross-sectional analysis of 20000 -patient database in Spain. J Hypertension. 2007;25:977-84.

21. Thijis L, Hansen T, Kikuya M, Björklund-Bodegard K, Li Y, Dolan $\mathrm{E}$, et al. The International Database of Ambulatory blood pressure in relation to Cardiovascular Outcome (IDACO). Blood Press Monit. 2007;12(4):255-62.
22. Sandoya E. Estado actual del monitoreo ambulatorio de presión arterial. Rev Urug Cardiol. 2013;28:273-84.

23. Qin $T$, Jiang $H$, Jiao $Y$, Ke $Y$, Sun $N$, Wang J, Zhu J. Ambulatory arterial stiffness index correlates with ambulatory pulse pressure but not dipping status in patients with grade I/grade 2 essential hypertension. J Int Med Res. 2014;42(6): 1323-34.

24. Schillaci G, Parati G, Pirro M, Pucci G, Mannarino M, Sperandini L, Mannarino E. Ambulatory Arterial Stiffness Index Is Not a Specific Marker of Reduced Arterial Compliance. Hypertension. 2007;49:986-91.

25. Lee HT, Lim YH, Kim BK, Won Lee K, Ung Lee J, Soo Kim K, et al. The Relationship between Ambulatory Arterial Stiffness Index and Blood Pressure Variability in Hypertensive Patients. Korean Circ J. 2011;41:235-40. 